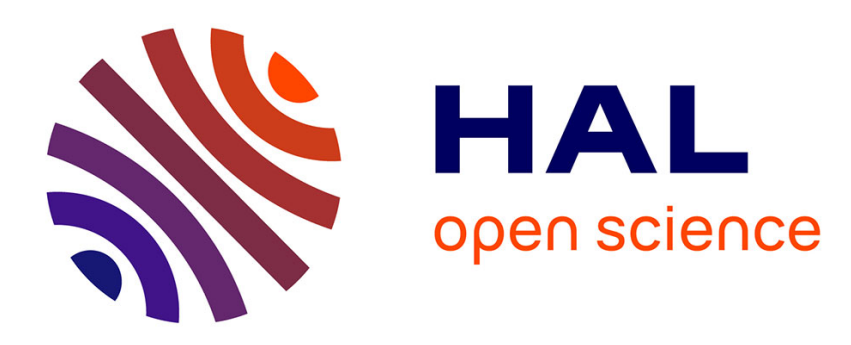

\title{
Ray-formed field scattering on the smooth localized surface in inhomogeneous media
}

\author{
A. Khil'Ko, I. Smirnov
}

\section{To cite this version:}

A. Khil'Ko, I. Smirnov. Ray-formed field scattering on the smooth localized surface in inhomogeneous media. Journal de Physique IV Proceedings, 1994, 04 (C5), pp.C5-1117-C5-1120. 10.1051/jp4:19945246 . jpa-00252934

\section{HAL Id: jpa-00252934 https://hal.science/jpa-00252934}

Submitted on 1 Jan 1994

HAL is a multi-disciplinary open access archive for the deposit and dissemination of scientific research documents, whether they are published or not. The documents may come from teaching and research institutions in France or abroad, or from public or private research centers.
L'archive ouverte pluridisciplinaire HAL, est destinée au dépôt et à la diffusion de documents scientifiques de niveau recherche, publiés ou non, émanant des établissements d'enseignement et de recherche français ou étrangers, des laboratoires publics ou privés. 


\title{
Ray-formed field scattering on the smooth localized surface in inhomogeneous media
}

\author{
A.I. KHIL'KO and I.P. SMIRNOV
}

Department of Hydrophysics and Hydroacoustics, Institute of Applied Physics, Russian Academy of Sciences, 46 Uljanov St., 603600 Nizhny Novgorod, Russia

\begin{abstract}
The effective method for the calculation of the acoustical field scattered by the spatially-localized smooth surfaces in weakly inhomogeneous media was developed. The method based on the solution of the problems of aiming the connected rays and calculation of the rays intensity by analytically determined curvatures of the transverse sections of the beams. The results of numerical calculation of the scattered field structure for the different displacements and orientations of the ellipsoid in the refractive layered medium are represented.
\end{abstract}

\section{INTRODUCTION}

The direct trajectories (rays) are usually used for the calculation of the scattering of the short wave fields by smooth-inhomogeneous surfaces in homogeneous media. In this case the intensity of scattering rays is determined by the curvatures of surfaces in the points of ray scattering. The solution of scattering problem in weakly inhomogeneous media becomes more difficult. On the one hand, we have the many curve rays and it is difficult to choose the all rays connecting the points where the source, receiver and the scattering point of surface are situated. On the other hand, the intensity of ray in weakly inhomogeneous media are depends on the coordinates of the observation point. Therefore should be used for the calculation of the scattered ray intensity both the ray fronts and scattering surface curvatures. Therefor, two problems should be solved for the calculation of the field scattered by smoothly-inhomogeneous spatially-localized surfaces in weakly inhomogeneous media. The first one, is the problem of aiming (the choice of all rays illuminating of the scatterer and forming the received field in the observation point after scattering). The second one, is the calculation of the scattered ray intensity. The effective method for the calculation of the scattered field in the weakly inhomogeneous media is developed in this investigation.

\section{SOLVING THE FIELD SCATTERING PROBLEM}

The scattered field calculation is based on the geometrical theory of diffraction (GTD) $\left(^{1}\right)$. Let the source point $S$, the point receiver $R$ and the rigid localized inhomogeneity $P$ (its size is less than the scale of the field variation) be placed in the smoothly inhomogeneous medium. The total received field can be written as: $u=\sum A_{n_{1}} e^{i k S_{n_{1}}}+k^{-1} \sum \vec{A}_{n_{2}} e^{i k S_{n_{2}}}+\left(k^{-1}\right)^{2}[\ldots]=u_{0}+u_{1}+\ldots$. The first part of $u_{0}$ describes the field as in the geometrical acoustics ( $A_{n}$ is the amplitude, $S_{n}$ is the phase and $k$ is the wave index). This group includes both the ordinary waves, propagating along the curve trajectories and the waves, reflected by the inhomogeneity. The second one, $u_{1}$ describes diffractional 


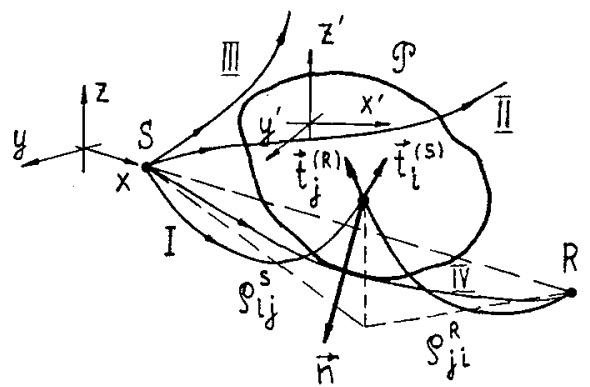

Figure 1: The disposition of the source $S$, the receiver $R$ and the scatterer $P$. Rays corresponding to reflected (I), refracted (II), diffractional (III) and sliding (IV) waves are shown.

amendments of the first order relative to $k^{-1}$. There are two subgroups in this group. The first one, describes the diffractional effects in the ordinary wave (the transverse amplitude diffusion, the diffraction on the bounds of the waveguide, etc.). The second one, is associated with the diffraction on the localized inhomogeneity and includes diffractional waves of different types.

\subsection{The problem of aiming}

It is necessary to trace curve trajectories (rays) in order to calculate fields, mentioned above. Looking over all rays can be made, but this method is often unfit because it requires the great amount of the calculation for small inhomogeneity. Approximate estimations (confirmed by the practice) show, that it grows proportionally to $x / R$, where $x$ is the length of the propagation route, $R$ is the scale of the inhomogeneity.

Special methods of aiming are more effective for small inhomogeneities $\left({ }^{2}\right)$. The one of these methods is described below. Outside of the inhomogeneity the ray submits to rules of geometrical acoustics (Fermat's principle). Thus the problem of aiming consists in selecting the pairs of rays coupling points $S$ and $R$ with the point of the scattering surface. These pairs must satisfy to conjugation conditions at given point of the surface. They are determined by the ray type.

For example, the conjugation condition for the wave (I) (see Fig.1), reflected from the inhomogeneity surface is: $\vec{N}=-\left(\vec{t}_{S}+\vec{t}_{R}\right) \sqrt{2}\left(1+<\vec{t}_{S}, \vec{t}_{R}>\right)^{-\frac{1}{2}}$, where $\vec{t}_{S, R}$ is a ray orts, drawn at the point of reflection, $\vec{N}$ is the external normal at this point, $\langle\ldots$,$\rangle is the scalar product of vectors.$

There is no universal algorithm of drawing orts of $\vec{t}_{S, R}$ at the point of contact for arbitrary inhomogeneous media and localized inhomogeneities. None the less, these orts can be approximated by $\vec{t}_{S, R}^{*}$ of rays connecting points $S$ and $R$ with fixed point $P^{*}$ of the inhomogeneity. It is possible if the size of the inhomogeneity is essentially less, than the scale of variations of the field, radiated by sources placed to the points $S$ and $R$ near the inhomogeneity. (It is correct inhomogeneities, placed far from caustic surfaces and waveguide bounds $\left({ }^{1}\right)$.) However, the general way of the solution doesn't change for taking these peculiarities into account. The procedure of aiming on the inhomogeneity can be summarized as follows: 1) tracing rays, coupling points $S, R$ with $P^{*} ; 2$ ) drawing ray orts $\vec{t}_{S, R}^{*}$ at this point; 3 ) searching the approximated normal or $\vec{e}^{*}$ at the contact point based on $\vec{t}_{S, R}^{*}$; 4) selecting points of the surface with found normals; 5) aiming on selected points and checking necessary conditions for each pair of rays. The parameters of rays become the next approximation, if these conditions are done.

Aiming on points of the surface of the small ellipsoid is discussed below. It is supposed, that the ellipsoid is acoustically homogeneous $n_{1}=n_{2}=n, \vec{\tau}_{1}=-\vec{\tau}_{2}$. The central point of the ellipsoid, called $P^{*}$, is the zero of the cartesian coordinate system. Coordinates of a point of the ellipsoid are expressed by components of the normal $\vec{N}$, drawn at this point: $\vec{r}=\vec{g}(\vec{N})=\left(a^{2} N_{x} \vec{i}+b^{2} N_{y} \vec{j}+\right.$ $\left.c^{2} N_{z} \vec{k}\right)\left(a^{2} N_{x}^{2}+b^{2} N_{y}^{2}+c^{2} N_{z}^{2}\right)^{-\frac{1}{2}}$, where $\vec{i}, \vec{j}, \vec{k}$ are the orts of cartesian coordinate system, $a, b, c$ are the half-sizes of the ellipsoid. The orts of rays, reached the point $P^{*}$ are called $\vec{t}_{S, R}^{*}$. For example, one can find the point of the surface, where the illuminating ray is reflected, using the formula 
for $\vec{N}: \vec{r}_{0} \simeq \vec{g}\left(-\left(\vec{t}_{S}^{*}+\vec{t}_{R}^{*}\right) \sqrt{2}\left(1+<\vec{t}_{S}^{*}, \vec{t}_{R}^{*}>\right)^{-\frac{1}{2}}\right)$. (The analogous aiming algorithm for refracted, diffractional and sliding waves can be used.)

The numerical experiments were done for bilinear waveguide. They demonstrated the practical fit of discussed algorithm. The iterrative process converges with geometrical speed, if the searched ray exists. The algorithm becomes circular, if there is no solution. This fact is easily noted. The time gain of aiming is inversely proportional to the ellipsoid size.

\subsection{Calculating field amplitudes}

The next stage of calculating the scattered field is searching amplitudes of waves, propagating along rays. In accordance with GTD these amplitudes can be found from formulae: $A_{d}=$ $A_{\text {ord }} S\left(\vec{t}_{S}, \vec{t}_{R}\right)|J|^{-1}$ where $A_{\text {ord }}$ is the amplitude of the ordinary wave, $J$ is the Jacobi's determinant of the transformation, which is proportional to the area of the transverse section of the beam, bringing the field from the point of falling to the receiver, $S($.$) is the diffractive index \left({ }^{3}\right)$. The fields of reflected and refracted waves are expressed analogously.

Let's consider, for example, the smooth curve surface, placed in horizontally stratified inhomogeneous waveguide (Fig. 1) and calculate the intensity of the wave, reflected from this surface. It is supposed the source $S$ is situated at the beginning of coordinate system. The ray coordinates are the azimuthal $\phi$ and polar $\theta$ angles of the ray orth and natural parameter $l$, which is equal to the length counted out along the ray to the current point $\vec{r}(\theta, \phi, l)$. This coordinate system is conformed to the form of the beam, but not with the form of the surface. The function $\vec{R}(M, \vec{\tau}, l) \equiv(X, Y, Z)$ , called the ray function describes the trajectory from the point $M(X, Y, Z)$ along the direction $\vec{\tau}=(\cos \beta \cos \gamma, \cos \beta \sin \gamma, \sin \beta)$. The derivative of this function must be calculated to trace the beam: $\vec{R}_{l}^{\prime}=\vec{t}, \vec{R}_{z}^{\prime}=\left(\vec{k}-t_{z} \vec{t}\right)\left(\frac{\partial z^{(\rho)}}{\partial z}-1\right), \vec{R}_{\beta}^{\prime}=\left(\vec{k}-t_{z} \vec{t}\right) \frac{\partial z^{(\rho)}}{\partial z}, \vec{R}_{\gamma}^{\prime}=\rho \vec{e}_{\gamma}$ where $\rho=\sqrt{x^{2}+y^{2}}$ is the ray orth at the current point, $e_{\gamma}=\frac{1}{\rho}(-Y, X, 0)$ is the vector orthogonal to the ray plane, $z^{(\rho)}$ is the vertical coordinate of the point of ray on the surface $\rho=$ const. Then the set of reflected rays is given by the formula:

$$
\vec{r}(\theta, \phi, l)=\vec{R}(\theta, \phi)+\vec{R}(P(\theta, \phi), \vec{\tau}(\theta, \phi), l-l(\theta, \phi))
$$

where $\vec{R}(\theta, \phi)=R\left(0, \vec{t}^{0}, l(\theta, \phi)\right)$ is the radius-vector of the point $P(\theta, \phi)$ of the ray reflection from the surface.

Let the wave (I) be reflected from the surface and reach the receiver. Then the intensity of this wave is (W means the power of the point source):

$$
I=|\Gamma|^{2} W(4 \pi)^{-1} t_{\perp}^{0}|D|^{-1}, D=\left\langle\vec{r}_{\theta}^{\prime},{\overrightarrow{r^{\prime}}}_{\phi}, \vec{r}^{\prime} l\right\rangle
$$

where $\Gamma$ is the Fresnel's coefficient at the reflection point and the expression of Jacobi's determinant $D$ can be derived from (1), (2), basing on Weingarten's derivation formulae and coefficients of 1st and 2 nd quadratic forms of the surface.

\section{SOME NUMERICAL RESULTS}

The method for quickly choice of the connected rays and increase of the accuracy of the ray intensity calculation was developed in this investigation. As it was showed above, for these aims we can use a prior information about the displacement of the scatterer and also analytical formulae for the calculation of the intensity of the scattered rays.

The efficiency of our method was demonstrated by the calculation of the acoustical fields, scattered by rigid ellipsoid in be-linear layered waveguide with following dependencies of sound velocity $(z[\mathrm{~km}] / c[k m . p . s]):.(0.0 / 1.500),(-0.2 / 1.470),(-3.0 / 1.550)$.

The results of calculation shows that the structure of the scattered fields is determined by displacement of ellipsoid relative to the source and receiver as well as axis and walls of the waveguide. The Fig. 2 shows the structure of scattered at the abservation point when the source and receiver are situated on the depth equal to $0.1 \mathrm{~km}$, and the distance between source and receiver is equal to $100 \mathrm{~km}$. The Fig. 2a represents the intensity variation when the ellipsoid is removed in horizontal 

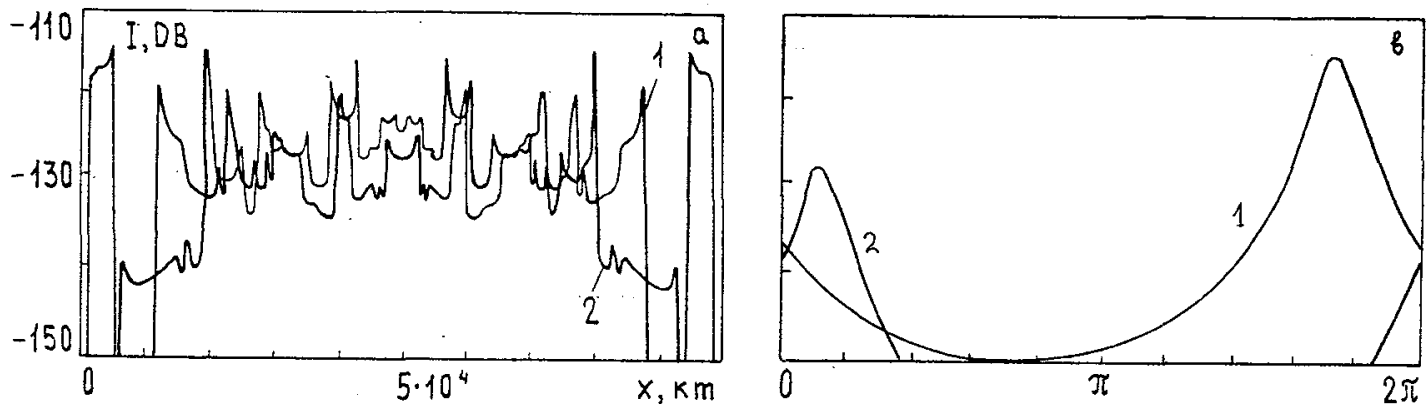

Figure 2: The dependence of the intensity of the field, scattered by the ellipsoid, on the its displacement (a) and orientation (b).

plane on direct trajectory between the source and receiver (curve 1) and on parallel trajectory on the same plane for the distance $1 \mathrm{~km}$ (curve 2). It can be seen that the intensity of scattered field is determined by shadow and focusing zones for the illuminated and received fields in the layered waveguide. The variations of the scattered field for the case when the orientation of the ellipsoid is changed are showed on the Fig $2 \mathrm{~b}$ for two stationary ellipsoid locations: $x=20 \mathrm{~km}, y=1 \mathrm{~km}$ (curve 1 ) and $x=25 \mathrm{~km}, y=1 \mathrm{~km}$ (curve 2).

\section{References}

[1] Kravtsov Yu.A and Orlov Yu.I. Geometrical Optics of Inhomogeneous media (Nauka, Moscow, 1980)

[2] Smirnov I.P. and Khil'ko A.I. Vesti KPI Electoacoustics and sound- techniques 16 (1992) 9-12

[3] Smirnov I.P. and Khil'ko A.I. Vesti KPI Electracoustics and sound- techniques 16 (1992) 6-9 